\title{
Study on the Experimental Teaching Mode of Probability and Statistics in Agriculture and Forestry University
}

\author{
Zhu Zhiyong*, Dong Enmei \\ College of Science, Northwest A \& F University, Yangling, China \\ Email address: \\ zhuzy1011@nwsuaf.edu.cn (Zhu Zhiyong), enmei117@sohu.com (Dong Enmei) \\ ${ }^{*}$ Corresponding author
}

\section{To cite this article:}

Zhu Zhiyong, Dong Enmei. Study on the Experimental Teaching Mode of Probability and Statistics in Agriculture and Forestry University. Science Innovation. Vol. 5, No. 1, 2017, pp. 51-56. doi: 10.11648/j.si.20170501.19

Received: February 7, 2017; Accepted: March 11, 2017; Published: March 15, 2017

\begin{abstract}
Probability theory and mathematical statistics is a subject with great applicability, and an important basic course required by almost all specialties in agriculture and forestry universities. To improve probability and statistics literacy of agricultural and forestry practitioners, cultivate innovative talents that can solve practical problems with professional background knowledge by using probability and statistics knowledge, we think that it is urgent to change the existing teaching mode, integrate the ideas of mathematics experiment into the teaching of probability theory and mathematical statistics, great attention must be paid to linking theory with practice.
\end{abstract}

Keywords: Agriculture and Forestry University, Probability and Statistics, Teching Mode, Mathematics Experiment

\section{农林院校概率统计课实验教学模式研究}

朱志勇 ${ }^{*}$ 董恩梅

理学院, 西北农林科技大学, 杨凌, 中国

邮箱

zhuzy1011@nwsuaf. edu.cn（朱志勇），enmei117@sohu. com（董恩梅）

摘要: 概率论与数理统计是一门应用性很强的课程, 是高等农林院校几乎所有专业必修的一门重要基础课。要提高农林 从业人员的概率统计素养, 培养能熟练应用概率统计知识解决带有专业背景实际问题能力的创新人才, 我们认为必须变 革现有的教学模式, 把数学实验的思想融入到概率论与数理统计的教学中去, 强化理论联系实际。

关键词: 农林院校, 概率论与数理统计, 教学模式, 数学实验

\section{1. 引言}

当前, 概率统计的思想和方法日益渗透到国民经济的 各个领域, 并广泛应用于自然科学、农学、林学、经济学、 金融保险甚至人文科学中。概率论与数理统计课程 (简称 概率统计课) 教学是农林院校本科数学教育中的一个重要 组成部分, 也是提高农林从业人员数学素养, 培养能熟练 应用概率统计知识解决带有专业背景实际问题能力创新
人才的一个重要的教学环节。这门课的教学内容是由来自 概率论和数理统计两个数学分支的基本知识内容组成。概 率论是研究和揭示随机现象统计规律性的一个数学分支, 而数理统计是以概率论为基础研究如何有效地收集整理、 分析受随机影响的数据, 并对所研究问题作出统计推断、 预测或者决策的另一个数学分支。概率论与数理统计课内 容丰富, 实践性强、应用性强, 其思想方法在培养大学生观 
察问题, 分析问题, 解决问题, 理论联系实际上都有着其 他数学课程不可替代的作用。

21世纪以来, 我国农林现代化发展步伐加快, 尤其是 加入WT0后，国际科技和贸易交流更加频繁，农林科技的 发展面临重大机遇和挑战, 对农林从业人员数学素养的要 求越来越高, 需要我们培养的农林从业人员不仅能通过专 业理论考试, 而且能够将知识转化为能力, 在实际工作中 发挥更大的创造性。目前, 针对 2010年7月13日 “全国教 育工作会议” 出台的《国家中长期教育改革和发展规划纲 要 (2010-2020) 年》, 以及教育部于 2012 年4月颁发的《教 育部关于全面提高高等教育质量的若干规定》，很多农林 院校提出了创新教育。面对农林院校的创新教育, 如何对 农林院校公共基础数学课的教学重新准确定位, 确立课程 的教学目标及其教学策略; 如何进一步改革课程的教学内 容和教学模式, 以服务于创新教育目标, 这些都是值得深 入探讨的问题。本文仅就农林院校一门很重要的本科数学 公共基础课一概率论与数理统计加以探讨。我们认为把数 学实验的思想和方法融入到农林院校各专业的数学课程 中去 (简称数学实验教学模式), 可以激发学生学习数学 的兴趣, 提高学生的数学理论水平和认知能力, 进而提高 学生解决实际问题的建模能力。

数学实验教学模式在我国还是一个新鲜事物, 其萌芽 也不过追溯到上个世纪 90 年代末, 在国际上, 从数学实验 课的首次出现到现在也仅有不到30年的时间。1997年，作 为数学公共基础课教学改革的产物, 国防科技大学首先开 始数学实验教学, 随后中国科技大学、北京大学、清华大 学、北京师范大学也陆续分别在1997年和1998年展开了数 学实验教学。据我所知, 在我国, “将数学实验的思想和 方法融入到各专业数学公共基础课的教学中去” 最早是由 北京理工大学叶其孝教授在文献 [15] 中提出的。此后, 近 14 年来, 在大学生数学建模竞赛的推动下, 数学实验教学 模式研究迅速高涨起来, 研究者发表了大量文章, 取得了 很多成果。然而, 正如刚才我们所说数学实验教学模式还 是一个新鲜事物, 从近三年取得的研究成果看 [1-7], 它 还没有形成完善的教学内容和教学体系, 很多学校也只是 在做一些尝试和探索, 改革中也遇到了不少的困难, 因此 我们有必要不断对其进行深入系统的研究、分析和总结。

\section{2. 数学实验的教学价值}

什么是数学实验, 目前没有统一的定义, 不同的学者 有不同的理解。在文献 [9], 作者是这样描述数学实验的, 数学实验就是为促进理性思维, 验证数学猜想, 归纳数学 规律, 获得数学知识, 解决数学问题, 通过一定的方法, 借助一定的设备, 运用一定的手段, 在数学思维活动的参 与下和典型的实验环境中所进行的一种数学建构过程和 数学探索活动。长期以来, 人们对数学的认识一直停留在 它是一门具有严谨系统的演绎科学, 数学活动只有高度的 抽象思维活动, 学习和研究数学只要有纸和笔就够了, 包 括许多数学工作者也这样认为, 认为物理, 化学和生物等 自然科学需要实验, 而数学不需要实验。但是, 历史证明, 数学不仅有逻辑证明, 还有实验。美籍匈牙利著名数学家
和数学教育家G. 波利亚 [16] 曾指出: “数学有两个侧面, 一方面它是欧几里得式的严谨科学, 从这个方面看数学象 是一门系统的演绎科学; 但另一方面, 创造过程中的数学, 看起来却象是一门试验性的归纳科学”。数学实验可以说 是自古有之, 并不是什么新鲜事物。人们从结绳计数开始 就在进行科学实验, 并通过实验不断的发展数学。传统的 数学实验是以测量、手工操作、制作模型、实物和教具演 示为主要形式, 以帮助学生理解概念、验证结论为主要目 的。随着计算机的普及和发展以及数学软件功能的越来越 强大, 改变了数学只能用笔和纸研究的方式, 特别是利用 计算机成功解决了 “四色问题” 对数学的研究方式方法产 生了巨大的影响。很多数学家开始着手创立一种新的学习 数学和研究数学的方法。例如, 我国著名数学家, 中国科 学院院士吴文俊先生发明的吴氏方法可在微机上很快地 证明困难的几何定理, 给定理机器证明的研究带来勃勃生 机。周咸青教授推广了吴氏方法并把它实现为有效的通用 程序, 证明了 512 条非平凡定理, 写成了英文专著。杨路 教授和中国科学院院士张景中则提出数值并行方法, 在低 档微机 (甚至计算器) 上实现了非平凡几何定理的机器证 明和机器发明。在这些数学家看来, 计算机的使用正在改 变着数学的性质, 数学正在变成一门 “实验科学”。从近 十多年来开设数学实验课取得的成绩看 $[8,10-15,17]$, 数 学实验课的开设至少起到了以下几个方面的作用: (1) 提高了学生学习数学的兴趣; (2) 激发了学生自己动手 解决实际问题的欲望; (3) 有助于学生独立思考、团队 意识和创新意识的培养; (4) 有助于学生掌握先进的数 学工具和计算机应用水平能力的提高; (5) 提高了学生 的综合素质。

\section{3. 概率统计课实验教学模式的组织实施}

（1）指导思想。这里我们倡导黑板+粉笔+多媒体课 件讲授方式为主数学实验为辅的指导思想。在整个教学内 容安排上分理论部分和实验部分, 学时按接近 $4: 1$ 的比例 分配。理论部分教学和实验部分教学交替进行, 而不是简 单的讲完 $4 / 5$ 学时的理论知识后, 剩下的 $1 / 5$ 学时进行实验 部分教学。黑板+粉笔+多媒体课件为主的传统教学方式, 对于理论教学有它不可替代的优点。所以, 理论部分教学 可以基本保持这种教学模式结构不变, 只是在一些学生理 解困难的知识内容上教师可根据需要增加一些演示实验, 一方面帮助学生理解所学知识, 另一方面又能帮助学生巩 固和熟悉数学实验的基本思想和方法, 丰富学生学习理论 知识的方法, 提高学习理论知识的兴趣, 激发学生动手解 决实际问题的欲望。例如, 在讲解蒙特霍尔问题时, 虽然 该问题答案在逻辑上没有问题, 但却十分违反直觉, 每次 讲到这个例子, 都会引发学生热烈的讨论。通常是教师绞 尽脑汁试着用不同的方式给学生解释, 但每次还是有相当 多的学生听不明白, 有时候甚至会忙中出错, 往往到最后 只能通过让学生自己课下再去争论而草草收场。这时教师 可以考虑少说多做, 在这个教学内容上增加一个演示实验, 模拟蒙特霍尔问题, 让学生切身体会产生蒙特霍尔问题的 游戏过程, 深刻去体会直觉不一定可信, 需要深入研究, 
实验可以提高我们的认识, 验证理论的正确性。实践证明, 这种做法让教师在解释蒙特霍尔问题时变的更容易和从 容了, 引发的争议少了, 学生接受起来更容易了, 课堂气 氛也更活跃了。在实验部分, 把教学内容也分为理论教学 和实践教学 (包括课内和课外)。抽出 4 个学时进行数学 软件的基础知识, 基本操作, 以及数学实验基本思想和方 法的理论教学。实践教学参照物理实验课和化学实验课的 上课方法, 但又有所不同, 不提前告知学生实验的结果, 主要让学生自己动手做实验, 观察得到结果, 然后分析结 果。实践教学不是追求内容的系统性和完整性, 而是以激 发学生的学习兴趣和提高解决实际问题的能力为主。

(2) 开设时间。最好安排在大学二年级下学期。选 择这个时间是因为这个时间农林院校各专业的学生基本 上都已开设过高等数学、线性代数和计算机课程, 已具备 进行概率统计理论课和实验课学习所需要的数学基础知 识和计算机基础编程知识。这个时间开课能有效保证整个 教学过程的顺利进行和流畅性。

（3）教学内容、学时安排和教学方法, 强化理论联 系实际。从多年教学实践来看, 目前农林院校概率统计课 课时数普遍偏少, 以我们所在的西北农林科技大学为例, 概率统计课只有 64 学时 (全为理论学时), 概率论教学与 数理统计教学各占 32 学时, 这一点与农业高校的专业要求 实际是不符的。我们时常见到有些学生虽获得第一手的实 验数据, 但由于缺乏统计知识而不能正确分析。我们认为 这和我们在有限的学时下讲的过急、过粗, 统计在实际问 题中的应用讲的太少, 学生实际动手演练的机会太少有一 定的关系。我们建议在增加总学时的同时压缩概率论讲授 的学时, 增加统计课时。总学时安排 76 学时为宜, 其中概 率论部分 32 学时 (理论 26 学时, 实验 6 学时), 数理统计 部分 44 学时 (理论 34 学时, 实验 10 学时)。在概率论的讲 授中, 注重概率论思想的讲解, 尽量避免抽象的理论证明, 以案例教学法为主, 通过对实际生活中典型案例的剖析解 释抽象的概念和原理, 说明思想, 以达到学生理解教学内 容的目的。例如, 在讲解随机变量的概念时, 我们可以先 不给出随机变量的概念, 先分别通过抛硬币和掷笁子的实 验告诉学生我们可以通过怎样的方式做到将实验结果数 量化, 随机变量本质是什么, 引入随机变量的目的是什么, 最后再给出随机变量的概念。此外, 也可在课堂上设计具 有典型性、针对性、启发性和趣味性的案例, 让学生参与 进来, 分析和互相讨论, 调动学生的主动性和积极性, 通 过引导鼓励学生提出解决问题的基本方法和途径。例如, 在讲二维连续随机变量函数分布之最大值和最小值分布 时, 我们可以设计一个金融上的价格竞拍问题: 某甲与其 它三人参与一个项目的竞拍, 价格高者获胜。若甲中标, 他就以 10 千美元转让给他人以牟利。可认为其它三人的竞 拍价是相互独立的，都服从 7 到 11 美元之间的均匀分布。 问甲应如何报价才能使自己获益的数学期望最大。通过这 个生动的案例让学生切实体会我们所学知识实用性, 激发 学生的学习兴趣、积极性和自信心。重视大数定律和中心 极限定理的讲解和理解, 因为这个内容是学习数理统计知 识的理论基础, 在统计学中用的很多, 尤其是统计中的近 似计算中更离不开它们。在数理统计的讲授中, 注重统计 思想的理解。以往教学中, 复杂公式的推导占用太多的课
时, 这实际上是不需要的, 这样做只会滋生学生的厌学情 绪, 对于今后学生的实际应用没有太多的帮助。可取的做 法是从复杂公式的推导中解脱出来, 注重基本概念、原理 的理解, 加强统计思想和统计方法的介绍, 以及在实际问 题中的应用。

（4）因材施教设计教学内容。理论部分, 不同专业 的学生所学的内容可以差别不大, 但在例题的选择上, 授 课教师需充分考虑到各专业的差别。考虑到理论部分教学 时数相比传统教学时减少不少, 所以需要根据学情精心选 择理论部分的教学内容或者重新编写一本教材。在实验部 分, 必须有一个适合不同专业学情的实验讲义教材, 以便 激发不同专业的学生自己动手和探索的兴趣。

（5）实践教学的实施。每次课内实践教学应该有一 个明确的目的, 要让学生知道要做什么, 怎么去做。此外, 什么时间上要合理, 要起到辅助理论教学的作用。在整个 课内实践教学的过程中, 教师主要起指导作用。每次实践 教学（2个学时90分钟）, 教师可以利用 15 分钟左右的时 间, 把这次试验的目的, 要求, 内容向学生交待清楚, 然 后剩下的时间交给学生。学生可以独自完成, 也可组队 (每 组人数不能多于 3 人) 通过讨论完成, 下课前 15 分钟向老 师报告实验过程及最终的结果。由老师现场对此次实验存 在的问题及主要创新点做出点评, 学生课后完成实验报告, 网上提交实验报告。课外实践教学主要以教师网上发布实 验作业, 学生在机房或宿舍独自或组队（要求同上）在规 定的时间内完成, 网上提交作业, 网上和教师进行互动为 主。

（6）考核办法。课程结束后的总评成绩由平时成绩、 期末考试成绩两部分组成。平时成绩占 $30 \%$, 在原有传统 打分方式的基础上增加平时的实验报告成绩（占平时成绩 的 50\%）。实验报告评分的基本标准是要自己动手, 写上 自己观察得到的结果, 写上自己的分析, 实事求是。期末 考试成绩由理论部分考试成绩和实验部分考试成绩构成, 各占 $50 \%$ 。理论部分考试按常规进行。实验部分考试在计 算机实验室进行, 考试内容包括基本问题, 验证性实验, 设计性实验和综合性实验, 用试卷答题 (实验方案、结果、 分析），网上提交试卷。

（7）师资队伍建设。概率统计课实验教学模式对任 课教师提出了更高的素质要求。数学实验思想和方法的融 入, 不仅要求教师应该具备扎实的理论课和实验课所需的 专业知识, 还需要教师对所授课学生的专业基础知识以及 这门课在所授课学生专业上的具体应用有个基本的了解。 因此必须通过有计划的、不间断的培训和进修, 引进相关 高学历、高水平人才等方式建立一支适合学情的高水平教 学团队来确保概率统计课实验教学模式实施的后劲和高 质量。

(8) 平台建设。先进的教学网络和专门的数学实验 室 (配备电脑和多媒体教学设备) 是保障概率统计课实验 教学模式顺利进行必不可少的硬件设施, 同时需有配套的 丰富数学软件支持, 如SPSS, Matlab、Mathematica、Maple 等。为了配合概率统计课实验教学模式, 在实验室宜建立 局域网, 与校园网对接, 便于学生在线播放教师的电子讲 稿和视频资源, 下载和上传学习资料, 在线讨论, 提交作 业、研发等; 老师可以发布作业, 上传资料, 发布消息, 
答疑, 检查学生实验进展情况等, 即需要建立服务器重点 支持的数学实验软件平台、使平台的设计能进一步加强老 师和学生对实验过程的参与, 具备更强的自主学习和研发 功能; 建立校园网支持下的网络教学平台, 使更多的学生 参与学习和主动学习、交互功能更强大。

\section{4. 概率统计课实验教学模式的实现}

根据以上改革思路, 我们设计了如图1所示的概率统 计课实验教学新模式。在此模式中, 教师在教室采用黑板 +粉笔+多媒体课件的讲授方式对概率统计课的理论部分 进行详细的讲解, 以及对实验部分的数学软件的使用, 数 学实验的思想和方法进行详细的介绍和课堂演示。课后,
除为帮助学生理解和消化课堂内容布置一定量的家庭作 业外, 教师还需根据教学内容精心设计不同难易程度的实 验题放到网上, 让学生在机房或者宿舍, 在规定的时间完 成这些实验作业。此外，教师也可以将自己手头与概率统 计课程有关的参考书, 优质视频资源, 相关网站的链接网 址, 有趣的课外知识, 典型案例, 科研课题等放在网上, 以拓宽学生的知识面, 激发学生的学习兴趣和提高学生的 参与度。通过网络以及学生提交的实验报告, 教师可以随 时和学生进行互动, 及时了解和掌握学生的学习情况, 给 予指导, 并及时对教学进度, 教学内容等作出相应的调整 以解决学生遇到的学习问题。

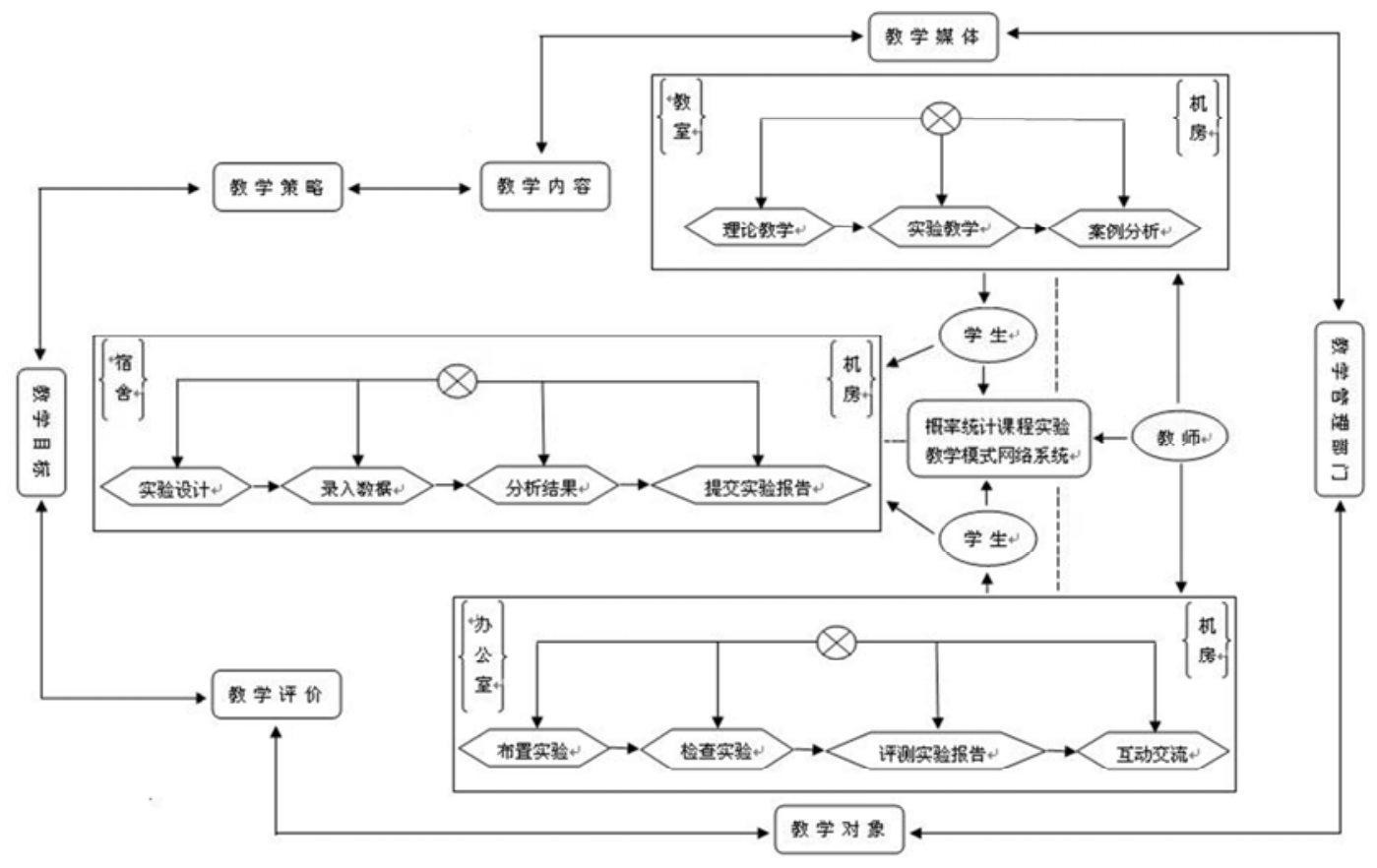

图1 概率统计课实验教学模式及其教学要素。

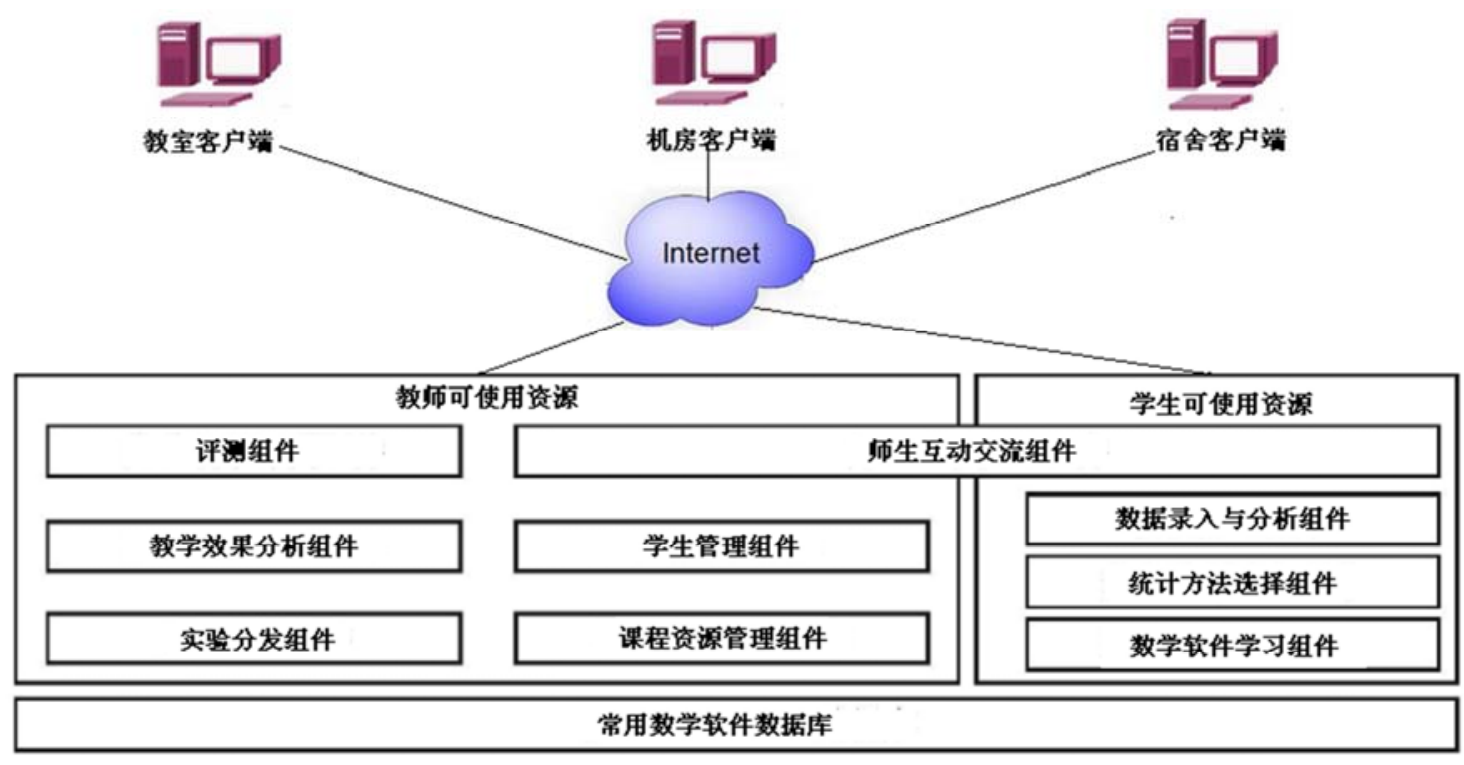

图2网络化概率统计课实验教学模式系统框架。 
SPSS, Matlab、Mathematica、Maple等常用数学软件 功能强大, 各有其优缺点。根据我们掌握的教学资源, 我 们在教学的实验部分采用以Matlab和SPSS统计软件为主, 其它数学软件为辅的教学策略。上课仅介绍Mat lab和SPSS 软件的使用, 而对学生做实验时数学软件的选择不加限制, 给学生充分的发挥空间。在此思路基础上, 我们提出了如 图2所示的基于各类常用数学软件的网络化概率统计课实 验教学模式系统框架。系统基于数学软件进行开发, 分别 提供教师和学生工具模块, 教师和学生通过网络访问系统。

\section{5. 概率统计课实验教学模式的效果}

为了检验我们提出的概率统计课实验教学模式的改 革效果, 我们在2016年春, 选择所在学校机械与电子工程 学院机化141-143班和车辆141-142班共5个班作为试点班 级 (简称实验班) 进行了试点教学, 并和 13 级未采用概率 统计课实验教学模式的对应班级 (简称非实验班) 进行了 横向比较。首先是通过问卷的方式分别对实验班和非实验 班的基本学情 (学习兴趣、学习态度、应用能力等) 在开 课前进行了调研, 得到如下表1所示结果:

表1 试点教学前基本学情调查。

\begin{tabular}{lllllll}
\hline \multirow{2}{*}{ 项目 } & \multicolumn{3}{l}{ 概率统计课的难易程度 } & \multicolumn{3}{l}{ 学习概率统计课的兴趣 } \\
\cline { 2 - 7 } & 难 & 中等 & 一般 & 感兴趣 & 一般 & 没兴趣 \\
\hline 实验班 & $55 \%$ & $34 \%$ & $11 \%$ & $36 \%$ & $55 \%$ & $9 \%$ \\
非实验班 & $57 \%$ & $35 \%$ & $8 \%$ & $38 \%$ & $49 \%$ & $13 \%$ \\
项目 & 认为概率统计课的实用性 & 对解决实际问题能力的提高 \\
实验班 & 很有用 & 一 一 般 & 没用 & 有帮助 & 一般 & 没帮助 \\
非实验班 & $83 \%$ & $16 \%$ & 2 & $35 \%$ & $57 \%$ & $8 \%$ \\
\hline
\end{tabular}

从表 1 我们可以看到, 超过 $80 \%$ 的学生认为概率论与数 理统计这门课很有用, 会对他们专业的学习有很大的帮助; 有近 $60 \%$ 的学生认为这门课很难学, 他们学习的压力很大; 有 $1 / 3$ 左右的学生对这门课感兴趣, 认为对提高他们解决 实际问题的能力有帮助。我们看到的是一个很矛盾的现象, 一方面学生觉得这门课很有用, 而另一方面学生却对这门 课的学习兴趣不高和认为对提高他们解决实际问题的能 力帮助不大。通过随机抽样和个别座谈的方式, 我们发现 了导致这一现象的根本原因是我校现有概率统计教学模 式只注重培养学生严密的逻辑推理能力和精细的计算能 力, 而忽视了对学生运用所学到的数学思想和方法解决实 际问题能力的培养。在现有教学模式下, 学生对数学的认 识也只是停留在记住了大量的计算公式、概念、定义、定 理和方法, 会照猫画虎做书本上的题而已, 而对书上这些 理论和方法为什么建立, 有什么用, 怎么用, 学生往往是 一头雾水, 知其然而不知其所以然。通过一个学期的试点 教学, 五个实验班（机化141-143和车辆141-142）期终考 试平均成绩分别为 83.3 分, 82.2分, 82.9分, 77.6 分, 71.2 分。每个班相比对应的非实验班 (机化 131-132和车辆 131-132) 的平均成绩 (分别为74.5分, 76.1分, 73.7分,
70.1分, 62.6分）平均高出7.6分左右。在整个教学过程 中, 我们看到的是实验班学生的学习兴趣要明显高于非实 验班, 更能主动积极地完成课后布置的作业 (理论课作业 和实验作业），在作业中也表现出更大的兴趣和创造性， 部分同学还对这门课进行实验教学模式改革的必要性和 如何改进提出了很多很好的意见。

试点教学后我们对实验班的学生又进行了问卷调查, 统计结果见表 2 。从统计结果中我们看到, 学生对这门课 的认可度提高了, 认为这门课难学的学生比例减少了近 20 个百分点, 改革初步取得了成效。随机走访学生了解到, 有的学生希望再少讲一点理论, 再多给他们留一些自己探 索的空间, 而有的学生则希望再多讲一些理论, 让他们有 更大的提升空间。通过这种教学模式让我们发现有学生希 望多学理论知识, 这是我们事前不曾想到的, 这是好事情。 但毕竟我们这门课学时有限, 正如文献[11]所提到的, 我 们的做法从其量只能算是 “开胃汤”, 而不是大餐, 将学 生学习数学理论知识的兴趣激发出来了, 胃口吊起来了, 我们这门课的目的就达到了。如果学生想学习更多的理论 知识, 学生可以去正规的 “餐馆” 去, 也可以通过看参考 书和利用网络上的视频资源满足其对于理论学习的渴望。 通过一个学期的教学, 我们也明显感觉虽然较以往的教学 模式, 工作量增加不少, 但教更有意思了, 更有挑战性了, 自己从中也收获不少, 课堂教学的氛围和效果更好了。

表2 试点教学效果调查。

\begin{tabular}{llllllll}
\hline \multicolumn{2}{l}{ 具有实用性 } & \multicolumn{2}{l}{ 学习兴趣提高 } & \multicolumn{2}{l}{ 能更好解决实际问题 } & \multicolumn{2}{l}{ 难易程度 } \\
\hline 是 & 否 & 是 & 否 & 是 & 否 & 难 & 其它 \\
$100 \%$ & $0 \%$ & $93 \%$ & $7 \%$ & $87 \%$ & $13 \%$ & $37 \%$ & $63 \%$ \\
\hline
\end{tabular}

\section{6. 结论}

总之, 数学类公共基础课实验教学模式还是一个新 生事物, 目前进行这方面尝试的农林院校还比较少, 在 我们学校也只是处于摸索和试点阶段, 虽取得一定的成 效和经验, 但课程的指导思想、上课内容和方式都有待 于在不断的试点中逐步明确, 这里还存在许多值得探讨 的问题和当前难以解决的问题, 如服务于实验教学模式 的软件平台的管理和日常维护, 师资资源的有效整合, 在开课学期, 学生其它课程负担过重, 授课人数过大等 问题。但我们相信, 随着现代计算机技术的不断发展和 数学软件功能的不断强大, 数学实验教学模式必然会展 现出自己强大的生命力。

\section{致谢}

本文为西北农林科技大学2015年教改项目数学专项 《“概率论与数理统计” 实验教学模式的探索与实践》 (JY1501001-1) 的阶段性成果之一。 


\section{参考文献}

[1] 刘雄伟. 基于Mathematica的高等数学教学过程研究与实践 [J]. 大学教育, 2016, 2:136-138。

[2] 孙蕾, 谷德峰. 概率论与数理统计实验教学案例设计 $[\mathrm{J}]$. 高等数学研究, 2014, 17 (1) : 100-103。

[3] 吴晓. 大学数学实验中的数学实验教学 $[\mathrm{J}]$. 大学教育, 2014 , $3: 116-117$ 。

[4] 刘超, 李尚志. 加强数学实验中的统计教学 $[J]$. 大学数学, 2013, 29 (4), 123-126。

[5] 张丽华. 高职电类专业数学实验教学改革探索 $[J]$. 教育探 索, 2013, 9:44-45。

[6] 谢治洲, 罗晓宾, 罗琼. 国内高校数学实验教学的现状分析 与研究 [J]. 教育与职业, 2012, 26:113-114。

[7] 叶军, 金栩. 由数学实验教学谈创新能力的培养 $[\mathrm{J}]$. 大学数 学, 2012, 28 (5) : 125-128。

[8] 韩明. 将数学实验的思想和方法融入大学数学教学 $[\mathrm{J}]$. 大 学数学, 2011,27 (4):137-141。

[9] 孙延洲. 试论数学实验的教学价值. 教育研究与实践 [J], 2010, 6:44-46。

[10] 丁卫平, 李新平. 基于数学实验的高等数学教学改革 $[\mathrm{J}]$. 高 等理科教育, 2007, 2:36-44。

[11] 李尚志, 陈发来, 张韵华, 吴耀华. 数学实验 $[M]$. 北京: 高 等教育出版社, 2004:1-249。

[12] 曹一鸣. 数学实验教学模式探究 $[J]$. 课程教材教法, 2003, $1: 46-48$ 。

[13] 李尚志, 陈发来. 《数学实验》课程建设的认识与实践 $[J]$. 数学的实践与认识, 2001,31 (6) :764-768。

[14] 盛中平, 王晓辉. 什么是数学实验 $[J]$. 高等理科教育, 2001, $2: 25-29$ 。

[15] 叶其孝. 把数学建模、数学实验的思想和方法融入高等数学 课的教学中去 $[J]$.工程数学学报, $2003,20(8): 3-13$ 。

[16] [美] G. 波利亚. 怎样解题 $[M]$. 阎育苏, 译. 北京: 科学出版设, 1982:1-231。

[17] 乐经良, 向隆万, 李世栋等著. 数学实验 $[\mathrm{M}]$. 北京: 高等教 育出版社, 1999: 1-227。 